\title{
Cross-sectional adherence with the multi-target stool DNA test for colorectal cancer screening in a large, nationally insured cohort
}

\author{
Lesley-Ann Miller-Wilson ${ }^{1}$ - Lila J Finney Rutten ${ }^{2} \cdot$ Jack Van Thomme $^{1} \cdot$ A Burak Ozbay ${ }^{1} \cdot$ Paul J Limburg ${ }^{2}$
}

Accepted: 16 May 2021 / Published online: 21 May 2021

(c) The Author(s) 2021

\begin{abstract}
Purpose Colorectal cancer (CRC) is the second most deadly cancer in the USA. Early detection can improve CRC outcomes, but recent national screening rates (62\%) remain below the $80 \%$ goal set by the National Colorectal Cancer Roundtable. Multiple options are endorsed for average-risk CRC screening, including the multi-target stool DNA (mt-sDNA) test. We evaluated cross-sectional mt-sDNA test completion in a population of commercially and Medicare-insured patients.

Methods Participants included individuals ages 50 years and older with commercial insurance or Medicare, with a valid mtsDNA test shipped by Exact Sciences Laboratories LLC between January 1, 2018, and December 31, $2018(n=1,420,460)$. In 2020, we analyzed cross-sectional adherence, as the percent of successfully completed tests within 365 days of shipment date. Results Overall cross-sectional adherence was $66.8 \%$. Adherence was $72.1 \%$ in participants with Traditional Medicare, $69.1 \%$ in participants with Medicare Advantage, and $61.9 \%$ in participants with commercial insurance. Adherence increased with age: $60.8 \%$ for ages $50-64,71.3 \%$ for ages $65-75$, and $74.7 \%$ for ages $76+$ years. Participants with mt-sDNA tests ordered by gastroenterologists had a higher adherence rate (78.3\%) than those with orders by primary care clinicians (67.2\%). Geographically, adherence rates were highest among highly rural patients (70.8\%) and ordering providers in the Pacific region (71.4\%). Conclusions Data from this large, national sample of insured patients demonstrate high cross-sectional adherence with the mt-sDNA test, supporting its role as an accepted, noninvasive option for average-risk CRC screening. Attributes of mt-sDNA screening, including home-based convenience and accompanying navigation support, likely contributed to high completion rates.
\end{abstract}

Keywords Colorectal cancer screening/prevention · Colorectal neoplasms · Early detection of cancer · Mt-sDNA · Insurance

\section{Introduction}

Colorectal cancer (CRC) is the second leading cause of cancer deaths and fourth most diagnosed cancer in the USA [1], representing an ongoing public health concern, with estimates of 149,500 incident and 52,980 fatal cases in 2021 [2]. Average-risk CRC screening can favorably impact the CRC public health burden by identifying patients with premalignant or localized malignant neoplasia for earlier, more effective intervention. National organizations such as the United States Preventive Services Task Force (USPSTF) in their
Paul J Limburg
limburg.paul@mayo.edu
Exact Sciences Corporation, Madison, WI, USA
2 Mayo Clinic, 200 First Street SW, Rochester, MN, USA

2021 Final Recommendation Statement and the American Cancer Society (ACS) recommend lowering the age for average-risk CRC screening (beginning at the age of 45 years) using one of several equally endorsed test options, including the multi-target stool DNA assay [3-5] (mt-sDNA; marketed as Cologuard®; Exact Sciences, Madison, WI). Since receiving approval from the US Food and Drug Administration in August 2014, the mt-sDNA assay has been prescribed by over 200,000 providers and completed by more than 4 million patients nationwide.

Between 2015 and 2018, estimated CRC screening rates increased overall by $4.2 \%$, from 61.7 to $65.9 \%$ according to National Health Interview Survey data [6,7]. The same National Health Interview Survey (NHIS) data showed that for those who completed screening, $4.1 \%$ used mt-sDNA, and use was consistent across demographic subgroups such as sex, age, and race/ethnicity, with no apparent 
disparities. The number of people screened with the mtsDNA test further increased in 2019, with 1.7 million patients successfully screened using mt-sDNA testing [8]. Despite the COVID-19 disruption, the at-home mt-sDNA test with its built-in navigation component for patients and providers and door-to-door shipping is uniquely positioned to sustain colorectal cancer screening efforts, potentially tempering the backlog of CRC screening delays and corresponding late-stage disease.

One critical component of effective CRC screening programs is patient adherence with completing the selected test option. The practical effectiveness of available screening strategies may be reduced by suboptimal adherence to screening recommendations [9]. Discouragingly, prior research has demonstrated relatively low completion rates for other stool-based CRC screening tests [9-14], and several recently conducted trials have revealed differences in CRC screening completion rates by test modality [9-11, 14]. These differences in completion rates may be exacerbated by multiple factors, such as socioeconomic status $[15,16]$ and race $[17,18]$. Given the differences between endoscopic, radiologic, and stool-based screening strategies, accurate understanding of test-specific patient adherence is critical for population-, provider-, and payor-level discussions.

To date, analyses of mt-sDNA adherence have demonstrated high cross-sectional adherence rates ( $71 \%$ ) but have been limited to Medicare beneficiaries to minimize the influence of insurance variability on test completion rates [19]. Given the growing adoption of mt-sDNA screening by commercial insurance plans, nearly all insured (>94\%) average-risk patients now have access to mt-sDNA screening with no out-of-pocket costs [20]. Thus, additional real-world assessment of mt-sDNA test adherence in nonMedicare patients is both feasible and timely. Here, we evaluated cross-sectional mt-sDNA test completion in a fully insured population and identified associated factors, to better inform shared decision-making, quality monitoring, and comparative effectiveness studies related to average-risk CRC screening.

\section{Methods}

Aggregate laboratory data from Exact Sciences Laboratories LLC (ESL; Madison, WI), the sole-source national laboratory for mt-sDNA testing, were retrospectively reviewed as part of ongoing laboratory quality management processes and in compliance with the Health Insurance Portability and Accountability Act. Per Mayo Clinic Institutional Review Board (IRB) criteria, this study was deemed as exempt from review.

\section{Study population, design, and data acquisition}

Eligible study participants included individuals who met the following criteria: ages 50 years and older, covered by commercial insurance or Medicare, with a valid mt-sDNA test shipped to the order-specified address from ESL between January 1, 2018 and December 31, 2018. At the time of the study, the USPSTF recommendation was to start screening at 50 years old. Valid mt-sDNA test shipments were defined as having all information required for ESL to analyze the sample and report a positive or negative test result. Deidentified data referent to available patient (sex, age), provider (specialty, practice location), and test order features (testing status, time to completion) were sourced from the ESL internal data systems. Of note, patient race/ethnicity is listed as an optional field on the mt-sDNA order form and was additionally collected when available.

Information from ESL internal data systems regarding order characteristics was initially collected for 1,508,087 patients. Inclusion and exclusion criteria (including provider/patient cancellations, duplicate orders, missing information, and reasons for ineligibility) were utilized to comprise the final analysis cohort consisting of 1,420,460 patients. The cohort attrition flow chart outlines that crosssectional adherence was defined as the percentage of eligible participants who successfully completed the test within 365 days of the shipment date (Fig. 1).

Sociodemographic characteristics including income and education are not tracked in the ESL test order data system. Therefore, these data were ascertained at the residential zip code level from public data sources, including the United States Census and American Community Surveys database [21-23].

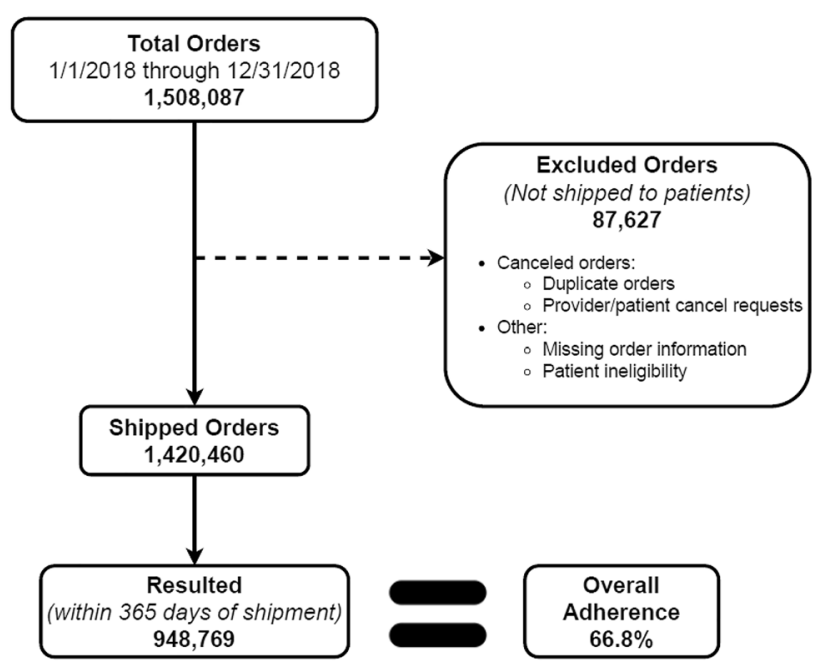

Fig. 1 Study cohort—attrition flowchart 


\section{Statistical analysis}

Descriptive statistics were used to describe the baseline characteristics of the study population overall and by insurance type. Counts and percentages were used to describe population-level statistics while distribution statistics (mean and median) were provided, where appropriate, to further describe the study populations' time to adherence, overall adherence, and age. Descriptive statistics were principally stratified by patient insurance type. Distribution statistics relating to skewness and other appropriate tests (e.g., chi-square test) were used as needed to describe the study population.

Given that the study population comprises the entirety of mt-sDNA patients during this time $(N=1,420,460)$, point estimates and $p$-value testing do not provide necessary context or support better comparison. In essence, very small differences, even if significant, are likely to arise due to the population size though do not reflect meaningful differences among populations. In contrast to significance tests, effect size is independent of sample size, while statistical significance can be dependent upon both sample size and effect size $[24,25]$. To avoid confounding the meaning of significance due to such a large sample size, we decided not to report two-sided $95 \%$ confidence intervals for point estimates or two-sided $p$-values for subgroup comparisons.

\section{Results}

Of 1,420,460 patients who met the study criteria (Fig. 1), $61.2 \%$ were female with a mean age of 64.9 years. Age distributions were 50-64 years $(47.3 \%), 65-75$ years $(40.0 \%)$, and $76+$ years $(12.7 \%)$, with the majority of the cohort residing in urban areas $(70.4 \%)$. Of those who specified a race/ethnic category, the majority of participants were white, although most participants $(85.6 \%)$ did not provide this optional information (Table 1). Within the study population, $46.1 \%$ had commercial insurance coverage, $33.5 \%$ had Traditional Medicare coverage, and $20.4 \%$ were covered by Medicare Advantage (Table 2). Patients residing in zip codes with above average Bachelor's degree attainment rates (35.0\% of all adults above 25 in the USA had a Bachelor's degree as of 2018) [22] accounted for $31.2 \%$ of all shipments. Patients in zip codes with a median income greater than the national median (\$33,706 per the American Community Survey conducted in 2018) [23] accounted for $43.3 \%$ of mt-sDNA test orders. Geographically, the highest adherence in the study population was observed for those dwelling in highly rural locations: urban (66.0\%), rural (68.5\%), and highly rural (70.8\%) (Table 3). Most mt-sDNA test orders were placed by primary care clinicians $(72.2 \%)$, followed by nurse practitioners and physician assistants (19.3\%), gastroenterologists (3.2\%), and obstetrics and gynecologists

Table 1 Study population demographics by insurance coverage type

\begin{tabular}{|c|c|c|c|c|}
\hline \multirow[t]{2}{*}{ Category ( $\%$ of cohort) } & \multicolumn{4}{|l|}{ Insurance type } \\
\hline & Commercial & Traditional Medicare & Medicare advantage & Total \\
\hline \multicolumn{5}{|l|}{ Age } \\
\hline $50-64, n(\%)$ & $573,126(87.5)$ & $56,953(12.0)$ & $41,501(14.3)$ & $671,580(47.3)$ \\
\hline $65-75, n(\%)$ & $71,187(10.9)$ & $307,007(64.5)$ & $190,617(65.8)$ & $568,811(40.0)$ \\
\hline $76^{+}, n(\%)$ & $10,546(1.6)$ & $112,111(23.5)$ & $57,412(19.8)$ & $180,069(12.7)$ \\
\hline Totals & 654,859 & 476,071 & 289,530 & $1,420,460$ \\
\hline \multicolumn{5}{|l|}{ Sex } \\
\hline Male, $n(\%)$ & $260,436(39.8)$ & $178,860(37.6)$ & $110,708(38.2)$ & $550,004(38.7)$ \\
\hline Female, $n(\%)$ & $394,174(60.2)$ & $296,962(62.4)$ & $178,732(61.7)$ & $869,868(61.2)$ \\
\hline Unspecified, $n(\%)$ & $249(0)$ & $249(0)$ & $90(0)$ & $588(0)$ \\
\hline \multicolumn{5}{|l|}{ Race } \\
\hline White $(\%)$ & $87,625(13.4)$ & $60,967(12.8)$ & $35,393(12.2)$ & $183,985(13.0)$ \\
\hline Black or African American (\%) & $6999(1.1)$ & $4304(0.9)$ & $4298(1.5)$ & $15,601(1.1)$ \\
\hline American Indian/Alaska Native (\%) & $244(0)$ & $176(0)$ & $103(0)$ & $523(0)$ \\
\hline Asian $(\%)$ & $2844(0.4)$ & $1034(0.2)$ & $820(0.3)$ & $4698(0.3)$ \\
\hline Native Hawaiian or other Pacific Islander (\%) & $190(0)$ & $119(0)$ & $79(0)$ & $388(0)$ \\
\hline Not specified (\%) & $556,957(85.0)$ & $409,471(86.0)$ & $248,837(86.0)$ & $1,215,265(85.6)$ \\
\hline \multicolumn{5}{|l|}{ Geography } \\
\hline Urban $(\%)$ & $471,962(72.1)$ & $317,426(66.7)$ & $210,140(72.6)$ & $999,528(70.4)$ \\
\hline Rural (\%) & $165,099(25.2)$ & $141,133(29.6)$ & $72,247(25.0)$ & $378,479(26.7)$ \\
\hline Highly rural (\%) & $17,798(2.7)$ & $17,512(3.7)$ & $7143(2.5)$ & $42,453(3.0)$ \\
\hline
\end{tabular}




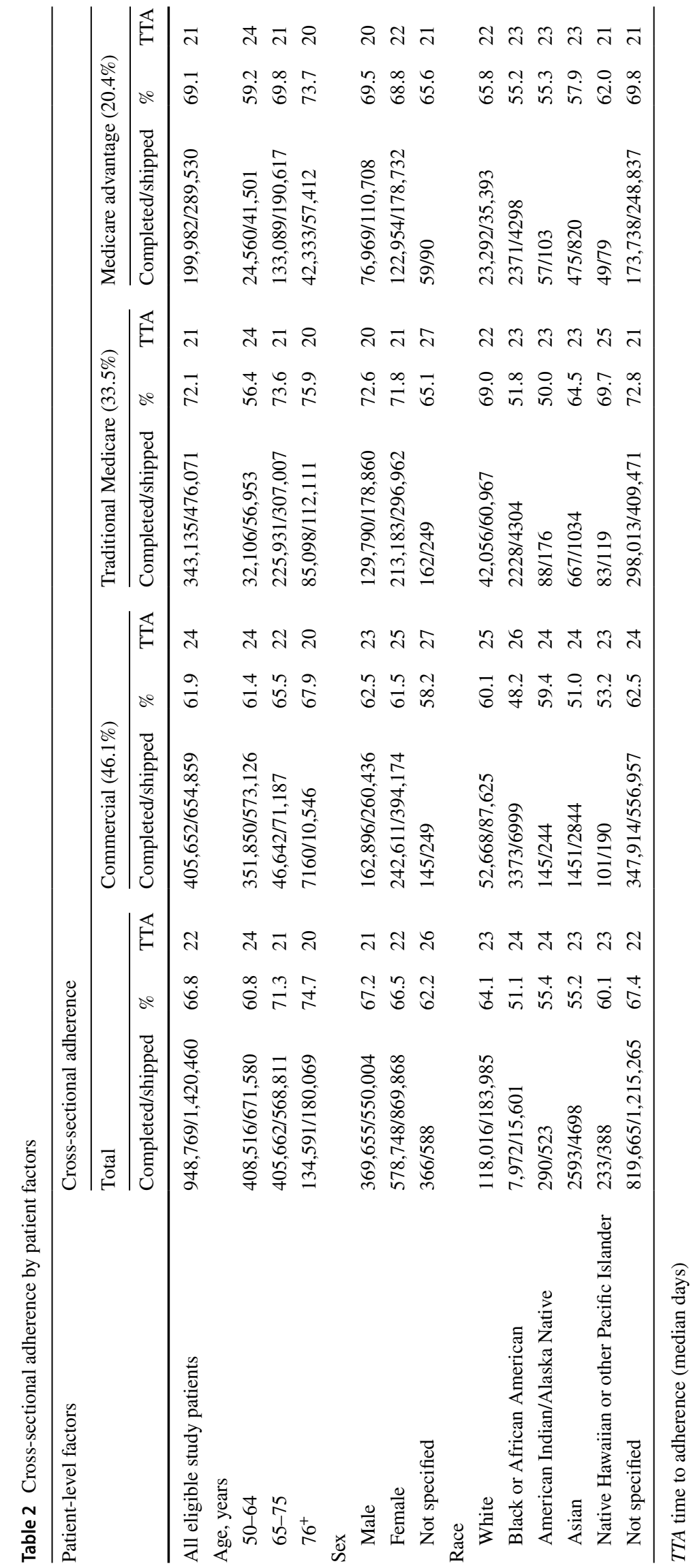




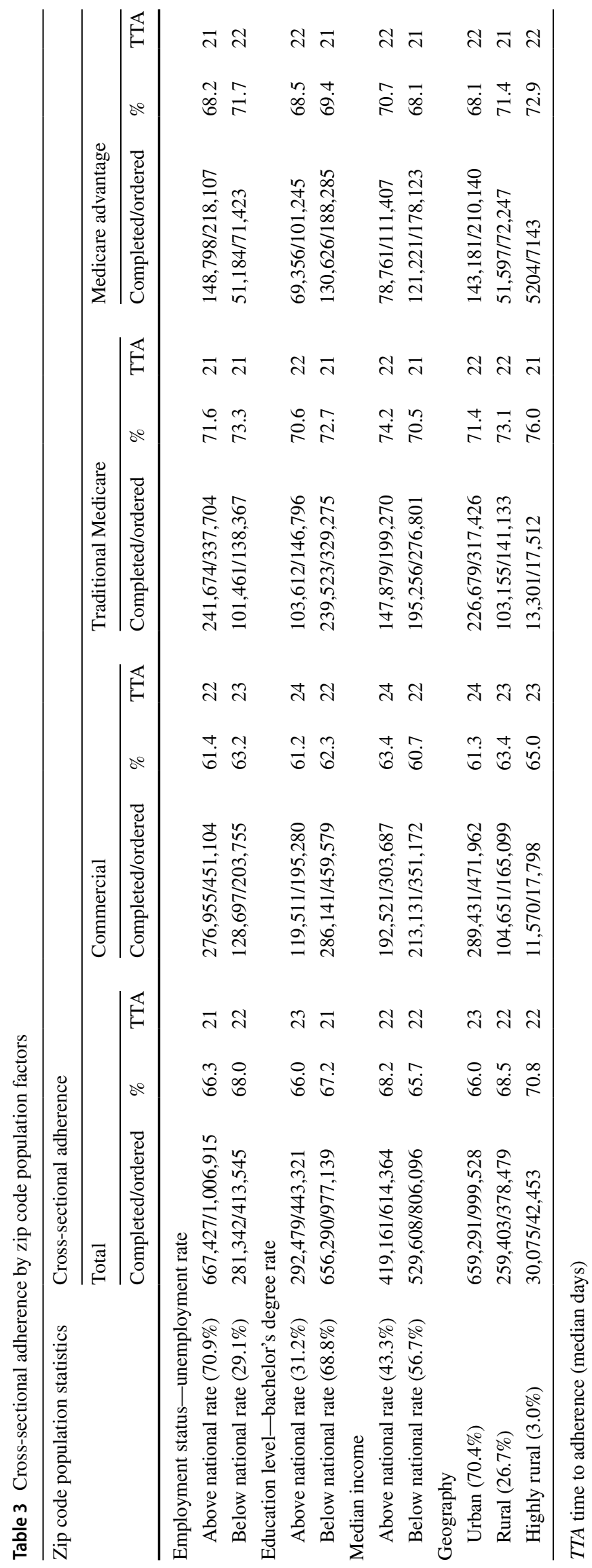


(1.8\%), with the remaining mt-sDNA test orders placed by other specialties (3.5\%) (Table 4).

The overall cross-sectional adherence rate for the entire study population was $66.8 \%$ (948,769/1,420,460 patients received and completed the mt-sDNA test and had a valid result) with a median time-to-adherence (TTA) of 22 days. The difference in adherence between males and females was small (67.2 vs. $66.5 \%$, respectively). Cross-sectional adherence was highest in patients with Traditional Medicare coverage $(72.1 \%)$ and lowest in patients with commercial insurance coverage (61.9\%). Adherence increased with age: $50-64$ years $(60.8 \%), 65-75$ years $(71.3 \%)$, and $76+$ years (74.7\%) overall, and within each of the insurance types (Table 2). Relative to those with commercial insurance, increases in adherence by age were more pronounced among those with Traditional Medicare or Medicare Advantage plans, who demonstrated lower rates of adherence among those 50-64 compared to those with commercial insurance (Table 2). Moreover, adherence was $73.6 \%$ for Traditional Medicare patients ages 65-75.

Statistically, cross-sectional adherence with the mt-sDNA test was not shown to be associated with level of education or median income of the zip code wherein patients resided. Adherence was $66.0 \%$ in zip codes with an above average Bachelor's degree attainment rate compared with $67.2 \%$ in zip codes with a below average Bachelor's degree attainment rate. Similarly, adherence was $68.2 \%$ compared to $65.7 \%$ in zip codes with median incomes above and below the national median, respectively (Table 3 ).

Adherence also varied among ordering provider specialty and practice location. Patients with mt-sDNA tests ordered by gastroenterologists exhibited a higher adherence rate $(78.3 \%)$ than those with orders placed by primary care clinicians $(67.2 \%)$, nurse practitioners and physician assistants (63.5\%), obstetricians and gynecologists (63.1\%), and other specialties (67.3\%). Adherence rates were highest among patients with ordering providers in the Pacific region (71.4\%) and West North Central region (70.1\%). Contrastingly, the lowest adherence was observed in the Mid-Atlantic region (65.7\%), New England (65.2\%), West South-Central region (64.6\%), and Puerto Rico/US Territories (60.7\%) (Table 4).

\section{Discussion}

Results from this retrospective study of a large, insured population demonstrate relatively high cross-sectional adherence with the mt-sDNA test for CRC screening. Adherence rates were similar between males and females and increased with age. Of note, area-level education and median income of patients' residential zip codes were not significantly associated with differences in adherence rates. Consistent with previous observations in Medicare patients [19], adherence rates were also higher for orders placed by gastroenterologists compared to other provider specialties. Geographically, the greatest adherence was observed among those living in highly rural areas; however, that population was only $3 \%$ of the overall cohort. Although GIs ordered the fewest mtsDNA tests, the rate of adherence to recommendations by disease specialists may relate in part to more detailed discussion during encounters regarding the importance of completing CRC screening; albeit, this speculative interpretation requires further evaluation.

With nearly 1.5 million participants included in the study population, our data contributes to the emerging literature regarding mt-sDNA test adherence in the clinical setting, although in a younger and larger cohort than seen previously [19]. Overall adherence approached $67 \%$, with meaningful differences observed between age groups. The highest rate was seen in the $76+$ years subgroup having Traditional Medicare coverage. Adherence also increased with age, from $60.8 \%$ in those $50-64$ to $74.7 \%$ in patients $\geq 76$ + years, while Traditional Medicare patients ages 65-75 exhibited $73.6 \%$ adherence.

The mt-sDNA test is offered in conjunction with patient and provider navigation support (available $24 \mathrm{~h}$ per day, 365 days per year, with translation services in over 240 languages). Previous studies have shown that patient navigation has a beneficial effect on patient health behavior, demonstrating increased CRC screening rates [19, 26, 27]. The amalgamation of test and navigation services was shown to be successful in a separate study of Medicare beneficiaries where individuals who were previously non-adherent with CRC screening were offered the mt-sDNA test, resulting in $88 \%$ completion and $96 \%$ diagnostic colonoscopy exam follow-up for those with a positive mt-sDNA result [28]. This intricate feature adds inherent value to the mt-sDNA test at no additional cost to the payor, health system, patient, or provider, while also improving the probability of test completion compared with no intervention [19-29]. This information may be helpful to consider as a guiding input variation for CRC modeling studies to investigate comparative effectiveness between screening modalities.

There is general agreement concerning the effectiveness of colorectal cancer screening; however, in contrast to other organ sites, major guideline groups have endorsed more than one test option for average-risk CRC screening in order to maximize engagement. Informed health care provider discussions and screening decisions are better guided by implementation considerations that can help patients select the testing option they are most likely to complete, and this factor is essential for reducing the number of CRC-related deaths in the USA. Prior research has demonstrated low completion rates for other CRC screening tests [9, 10], and existing literature on repeat screening with other non-mt-sDNA 


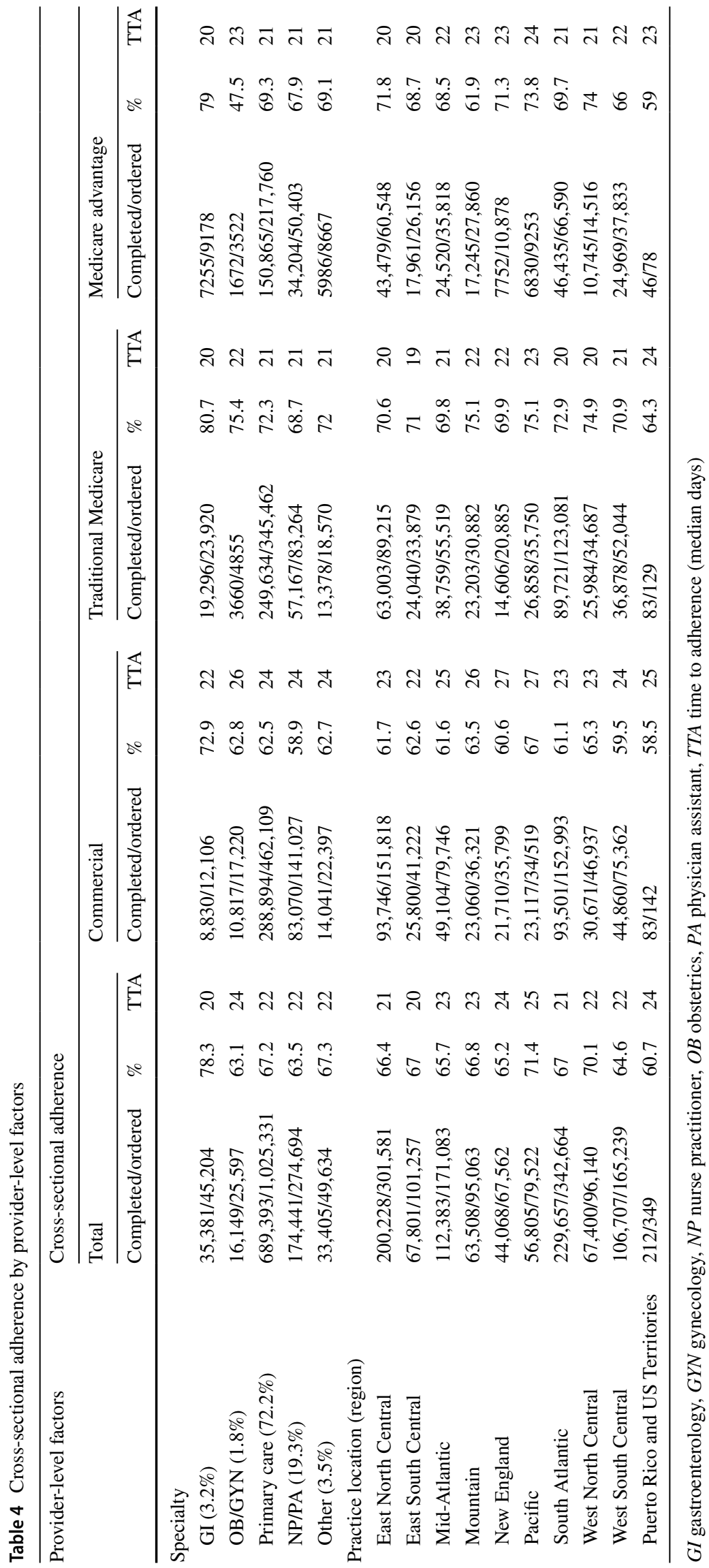


stool-based screening tests suggests a need for improvement [9-11, 30]. A more sensitive test, such as mt-sDNA, is less susceptible to drops in adherences rates. Mt-sDNA has a higher single application sensitivity for all stages of CRC, which distinguishes it from single marker fecal occult blood tests (FIT/gFOBT) [31]. The 10,000-person mt-sDNA pivotal study by Imperiale et al. [31] reported $92 \%$ sensitivity for CRC (vs $74 \%$ for FIT). This performance difference held true in receiver operating characteristic (ROC) analysis, regardless of the cutoff chosen for FIT. Higher one-time test sensitivity is relevant given that FIT adherence to recommended screening frequency can drop off substantially after the first cycle of annual testing [32].

As an integral part of the screening process, the follow-up colonoscopy to a positive non-colonoscopy CRC screening exam is becoming increasingly appreciated in the literature. Follow-up colonoscopy after a positive first-line test is necessary for complete and effective CRC screening [33, 34]. Failure to, and delay of follow-up after a positive stool-based test has been associated with increased risk of later stage CRC and CRC mortality [33, 35-37]. Doubeni et al. found that patients failing to follow-up on abnormal FIT results face a sevenfold higher risk of dying of CRC than those who complete the follow-up process [37].

Differences have also been revealed in CRC screening completion rates by modality $[13,14]$. In a study by Rutten et al. [14] among residents of Olmsted County, MN, eligible and due for CRC screening, it was revealed that CRC screening incidence rates remained stable from 2016 to 2018, while test-specific rates for mt-sDNA significantly increased. Moreover, the study revealed that adherence with follow-up colonoscopy within 6 months after a positive stool-based test was significantly higher among patients who underwent mt-sDNA screening versus FIT/FOBT (84.9\% vs $42.6 \%$, respectively). At real-world (imperfect) adherence rates of $40 \%$ for annual FIT and $70 \%$ for triennial mt-sDNA derived from a critical assessment of meta-analyses and retrospective cross-sectional data $[19,38]$ (in systems using FIT without a navigation program) [39], modeling illustrates that the number of LYG and reductions in CRC incidence and mortality were higher for triennial mt-sDNA [40].

Several geographic variations in adherence aligned with the currently reported US percentage of adults being up-todate with CRC screening tests by state [41], with the highest rates seen in the Pacific and West North Central regions in our study, although adherence was lower in the New England and Middle Atlantic regions where up-to-date screening hovers around $\geq 70 \%$ and $\geq 65 \%$, respectively.

Additionally, practice specialty was associated with higher cross-sectional adherence. Although gastroenterologists ordered fewer tests, adherence was lower among primary care providers. The observed difference in adherence by specialty could be related to various provider factors (strength of screening recommendation, patient perception of specialty-related knowledge), patient factors (attitude concerning referral/follow-up completion), or a combination. It is also likely that patients who present to a gastroenterologist bring with them specific concerns which may serve as motivation to screen. Although test adherence rates differed by practice, recent literature has shown widespread awareness of the 2018 ACS CRC guidelines among primary care providers [42]. A 2020 survey study by the Montana Cancer Control Program revealed that fecal DNA testing has become much more popular since 2016 , with more than a third $(35.2 \%)$ of primary care providers reporting discussing it with patients in 2020 compared to only 4\% in 2016 [43]. The same report from the Montana Department of Public Health and Human Services highlighted that in 2020 the fecal DNA test was the 3 rd highest ranked CRC screening test prescribed with $86 \%$ of ordering providers considering the test effective. Future CRC screening studies can be tailored to garner information on provider ordering characteristics to better inform screening strategy, assess prescription differences, and develop provider education initiatives.

We would like to acknowledge several limitations of our study. First, there was limited reporting of ethnicity in this study population, with less than $15 \%$ of patients specifying a racial category. The unavailability of such data prohibited us from examining an important demographic aspect associated with screening adherence; thus, the differences observed among race/ethnicity were not overly considered due to a large percentage of participants not providing the optional information. Patient and provider factors analyzed in our study were limited to those characteristics that could be captured from the existing laboratory database. A more detailed assessment of factors suspected to influence mtsDNA screening adherence, such as socioeconomic status along with a closer look at social determinants of health, will benefit future investigations. Secondly, we relied on determination of the mt-sDNA ordering clinician to define CRC average-risk status. Although indicated for mt-sDNA, we assumed that each provider appropriately followed test eligibility indications before prescribing, and we did not have access to sufficient data to confirm risk status for all participants in this real-world study. Thus, a percentage of off-label use can be logically assumed. Lastly, given the relative recency of mt-sDNA availability and adoption in clinical practice, we did not assess longitudinal adherence for the current study. Mt-sDNA test adherence was only evaluated on a cross-sectional basis with a review of associated patient and provider factors over a 1-year study period. Further evaluation of longitudinal adherence with triennial mt-sDNA screening is anticipated in future studies. 


\section{Conclusions}

Novel data from this large retrospective cohort of both Medicare and commercially insured patients revealed favorable adherence to CRC screening with the mt-sDNA assay test among eligible patients who met the study criteria, having a valid mt-sDNA test shipped to their orderspecified address from ESL between January 1, 2018, and December 31, 2018. Innovative features of the $\mathrm{mt}$ sDNA test such as the accompanying patient navigation system, together with its noninvasive approach and feasibility, likely impacted test completion rates. The highest adherence rates were observed among those covered by Medicare and for tests ordered by gastroenterologists. Our findings contribute valuable real-world data to the existing evidence base and provide clinical decisionmakers with supportive information to recommend a guideline-endorsed CRC screening strategy, with the goal of increasing adherence through test completion. Future investigations examining longitudinal completion rates in accordance with national guidelines, as well as more detailed analyses of associations with socioeconomic, race/ethnicity, and other demographic factors on mt-sDNA test completion rates, are anticipated to complement this study.

Acknowledgements Medical writing and editorial support were provided by Rebecca K Swartz, PhD, a former employee of Exact Sciences (Madison WI), and William K Johnson, $\mathrm{PhD}$, an employee of Exact Sciences (Madison, WI).

Author contribution Conceptualization-Lesley-Ann Miller-Wilson, Lila J Finney Rutten, Paul J Limburg, Ahmet Burak Ozbay. Data curationJack Van Thomme. Formal analysis-all authors. Investigation-LesleyAnn Miller-Wilson, Jack Van Thomme. Methodology—all authors. Project administration-Lesley-Ann Miller-Wilson, Jack Van Thomme. Resources-Lesley-Ann Miller-Wilson, Jack Van Thomme, Paul J Limburg. Software-Jack Van Thomme. Supervision—Lesley-Ann Miller-Wilson, Paul J Limburg. Validation—Lesley-Ann Miller-Wilson, Jack Van Thomme. Visualization-Lesley-Ann Miller-Wilson, Jack Van Thomme. Writing (original draft)—all authors. Writing (review \& editing)—all authors.

Funding This study was funded by Exact Sciences.

Availability of data and materials Study data are available upon reasonable request.

\section{Declarations}

Ethics approval The data herein were retrospectively reviewed as part of ongoing laboratory (Exact Sciences Laboratories, LLC) quality management processes in compliance with the Health Insurance Portability and Accountability Act (HIPAA). The study was deemed exempt from IRB review by the Mayo Clinic Institutional Review Board.

Consent to participate/consent for publication Not applicable; retrospective review of deidentified data.
Disclosure LAMW, JVT, and BO are employees and shareholders of Exact Sciences. LJFR offers scientific input to research studies through a contracted services agreement between Mayo Clinic and Exact Sciences. PJL serves as Chief Medical Officer for Screening at Exact Sciences through a contracted services agreement with Mayo Clinic. PJL and Mayo Clinic have contractual rights to receive royalties through this agreement.

Conflict of interest The authors declare no competing interests.

Open Access This article is licensed under a Creative Commons Attribution 4.0 International License, which permits use, sharing, adaptation, distribution and reproduction in any medium or format, as long as you give appropriate credit to the original author(s) and the source, provide a link to the Creative Commons licence, and indicate if changes were made. The images or other third party material in this article are included in the article's Creative Commons licence, unless indicated otherwise in a credit line to the material. If material is not included in the article's Creative Commons licence and your intended use is not permitted by statutory regulation or exceeds the permitted use, you will need to obtain permission directly from the copyright holder. To view a copy of this licence, visit http://creativecommons.org/licenses/by/4.0/.

\section{References}

1. American Cancer Society (2020) ACS Cancer Facts \& Figures. https://www.cancer.org/content/dam/cancer-org/research/cancerfacts-and-statistics/annual-cancer-facts-and-figures/2020/cancerfacts-and-figures-2020.pdf. Accessed 16 Nov 2020

2. Siegel RL, Miller KD, Fuchs HE, Jemal A (2021) Cancer Statistics, 2021. CA Cancer J Clin 71(1):7-33. https://doi.org/10.3322/ caac. 21654

3. Bibbins-Domingo K et al (2016) Screening for colorectal cancer: US preventive services task force recommendation statement. JAMA - J Am Med Assoc 315(23):2564-2575. https://doi.org/ 10.1001/jama.2016.5989

4. American Cancer Society (2020) Colorectal cancer guideline how often to have screening tests. https://www.cancer.org/cancer/colonrectal-cancer/detection-diagnosis-staging/acs-recommendations. html. Accessed 03 April 2021

5. Davidson $\mathrm{KW}$ et al (2021) Screening for colorectal cancer. JAMA 325(19):1965. https://doi.org/10.1001/jama.2021.6238. Accessed May 2021

6. U.S. Department of Health \& Human Services (2015) NHIS 2015 data release. National Center for Health Statistics. https:// www.cdc.gov/nchs/nhis/nhis_2015_data_release.htm. Accessed 05 Feb 2020

7. U.S. Department of Health \& Human Services (2018) NHIS 2018 data release. National Center for Health Statistics. https:// www.cdc.gov/nchs/nhis/nhis_2018_data_release.htm. Accessed 05 Feb 2020

8. Exact Sciences Corporation LLC (2020) United States Securities And Exchange Commission (Form 10-K) Annual Report Pursuant To Section 13 Or 15(D) Of The Securities Exchange Act Of 1934; Transition Report Pursuant To Section 13 Or 15(D) Of The Securities Exchange Act Of 1934. Accessed 12 Nov 2020. [Online]. Available: http://d18rn0p25nwr6d.cloudfront.net/CIK0001124140/32330bf1-f821-4b65-a147-638835b3a7e3.pdf

9. Singal AG et al (2017) Effect of colonoscopy outreach vs fecal immunochemical test outreach on colorectal cancer screening completion a randomized clinical trial. JAMA - J Am Med Assoc 318(9):806-815. https://doi.org/10.1001/jama.2017.11389 
10. Cyhaniuk A, Coombes ME (2016) Longitudinal adherence to colorectal cancer screening guidelines - PubMed. Am J Manag Care. https://pubmed.ncbi.nlm.nih.gov/26885670/. Accessed 17 Nov 2020

11. Liang PS et al (2016) Adherence to competing strategies for colorectal cancer screening over 3 years. Am J Gastroenterol 111(1):105-114. https://doi.org/10.1038/ajg.2015.367

12. Inadomi JM et al (2012) Adherence to colorectal cancer screening: a randomized clinical trial of competing strategies. Arch Intern Med 172(7):575-582. https://doi.org/10.1001/archinternmed.2012.332

13. Segnan $\mathrm{N}$ et al (2005) Randomized trial of different screening strategies for colorectal cancer: patient response and detection rates. $\mathrm{J}$ Natl Cancer Inst 97(5):347-357. https://doi.org/10.1093/jnci/dji050

14. Rutten LJF et al (2020) Colorectal cancer screening completion: an examination of differences by screening modality. Prev Med Rep 20. https://doi.org/10.1016/j.pmedr.2020.101202

15. Vutien P, Shah R, Ma K, Saleem N, Melson J (2019) Utilization of census tract-based neighborhood poverty rates to predict nonadherence to screening colonoscopy. Dig Dis Sci 64(9):25052513. https://doi.org/10.1007/s10620-019-05585-8

16. Blair A, Gauvin L, Ouédraogo S, Datta GD (2019) Area-level income disparities in colorectal screening in Canada: evidence to inform future surveillance. Curr Oncol 26(2):e128-e137. https:// doi.org/10.3747/co.26.4279

17. Daskalakis C, DiCarlo M, Hegarty S, Gudur A, Vernon SW, Myers RE (2020) Predictors of overall and test-specific colorectal cancer screening adherence. Prev Med (Baltim) 133. https://doi. org/10.1016/j.ypmed.2020.106022

18. Ni K et al (2020) Low colorectal cancer screening uptake and persistent disparities in an underserved urban population. Cancer Prev Res 13(4):395-402. https://doi.org/10.1158/1940-6207. CAPR-19-0440

19. Weiser E et al (2020) Cross-sectional adherence with the multitarget stool DNA test for colorectal cancer screening: real-world data from a large cohort of older adults. J Med Screen. https://doi. org/10.1177/0969141320903756

20. Exact Sciences Corporation LLC (2020) Support \& Coverage I Cologuard ${ }^{\circledR}$ For HCPs. https://www.cologuardtest.com/hcp/ coverage-and-support/coverage. Accessed 17 Nov 2020

21. United States Census Bureau (2018) 2018 data release new and notable. American Community Survey (ACS). https://www.census.gov/programs-surveys/acs/news/data-releases/2018/release. html\#par_textimage_copy. Accessed 18 Nov 2020

22. United States Census Bureau (2019) Number of people with master's and doctoral degrees doubles since 2000. America Counts: Stories Behind The Numbers. https://www.census.gov/library/stories/2019/ 02/number-of-people-with-masters-and-phd-degrees-double-since2000.html. Accessed 18 Nov 2020

23. Semega J, Kollar M, Creamer J, Mohanty A (2018) Income and poverty in the United States: 2018. United States Census Bureau. https://www.census.gov/data/tables/2019/demo/income-poverty/ p60-266.html. Accessed 18 Nov 2020

24. Sullivan GM, Feinn R (2012) Using effect size—or why the P value is not enough. J Grad Med Educ 4(3):279-282. https://doi. org/10.4300/jgme-d-12-00156.1

25. Ellis PD (2009) Thresholds for interpreting effect sizes. https:// www.polyu.edu.hk/mm/effectsizefaqs/thresholds_for_interpreting effect_sizes2.html. Accessed 18 Nov 2020

26. Rice $\mathrm{K}$ et al (2017) Increasing colonoscopy screening in disparate populations: results from an evaluation of patient navigation in the New Hampshire Colorectal Cancer Screening Program. Cancer 123(17):3356-3366. https://doi.org/10.1002/cncr.30761

27. Dougherty MK et al (2018) Evaluation of interventions intended to increase colorectal cancer screening rates in the United States: a systematic review and meta-analysis. JAMA Intern Med 178(12):16451658. https://doi.org/10.1001/jamainternmed.2018.4637
28. Prince M, Lester L, Chiniwala R, Berger B (2017) Multitarget stool DNA tests increases colorectal cancer screening among previously noncompliant Medicare patients. World J Gastroenterol 23(3):464-471. https://doi.org/10.3748/wjg.v23.i3.464

29. Champion VL et al (2018) A randomized trial to compare a tailored web-based intervention and tailored phone counseling to usual care for increasing colorectal cancer screening. Cancer Epidemiol Biomarkers Prev 27(12):1433-1441. https://doi.org/ 10.1158/1055-9965.EPI-18-0180

30. Gellad ZF et al (2011) Longitudinal adherence to fecal occult blood testing impacts colorectal cancer screening quality. Am J Gastroenterol 106(6):1125-1134. https://doi.org/10.1038/ajg. 2011.11

31. Imperiale TF et al (2014) Multitarget stool DNA testing for colorectal-cancer screening. N Engl J Med 370(14):1287-1297. https://doi.org/10.1056/nejmoa1311194

32. Murphy CC, Halm EA, Skinner CS, Balasubramanian BA, Singal AG (2020) Challenges and approaches to measuring repeat fecal immunochemical test for colorectal cancer screening. Cancer Epidemiol Biomarkers Prev 29(8):1557-1563. https://doi.org/ 10.1158/1055-9965.EPI-20-0230

33. Corley DA et al (2017) Association between time to colonoscopy after a positive fecal test result and risk of colorectal Cancer and Cancer stage at Diagnosis. JAMA - J Am Med Assoc 317(16):1631-1641. https://doi.org/10.1001/jama.2017.3634

34. Martin J et al (2017) Reasons for lack of diagnostic colonoscopy after positive result on fecal immunochemical test in a safety-net health system. Am J Med 130(1):93.e1-93.e7. https://doi.org/10. 1016/j.amjmed.2016.07.028

35. Tørring ML, Frydenberg M, Hamilton W, Hansen RP, Lautrup MD, Vedsted P (2012) Diagnostic interval and mortality in colorectal cancer: U-shaped association demonstrated for three different datasets. J Clin Epidemiol 65(6):669-678. https://doi.org/10. 1016/j.jclinepi.2011.12.006

36. Thomson CS, Forman D (2009) Cancer survival in England and the influence of early diagnosis: what can we learn from recent EUROCARE results? Br J Cancer 101(Suppl 2):S102-S109. https://doi.org/10.1038/sj.bjc.6605399

37. Doubeni CA et al (2019) Modifiable failures in the colorectal cancer screening process and their association with risk of death. Gastroenterology 156(1):63-74.e6. https://doi.org/10.1053/j.gastro.2018.09.040

38. Hassan C et al (2012) Meta-analysis: adherence to colorectal cancer screening and the detection rate for advanced neoplasia, according to the type of screening test. Aliment Pharmacol Ther 36(10):929-940. https://doi.org/10.1111/apt.12071

39. Piscitello A et al (2020) Estimating the impact of differential adherence on the comparative effectiveness of stool-based colorectal cancer screening using the CRC-AIM microsimulation model. bioRxiv. https://doi.org/10.1101/2020.08.30.271858

40. Fendrick M et al (2020) Sa1658 microsimulation study of lifeyears gained from screening versus follow-up colonoscopy using the CRC-AIM model. Gastroenterol AGA Abstr 158(6):S-372. https://doi.org/10.1016/S0016-5085(20)31623-1

41. Joseph DA, King JB, Richards TB, Thomas CC, Richardson LC (2018) Use of colorectal cancer screening tests by state. Prev Chronic Dis 15(6). https://doi.org/10.5888/pcd15.170535

42. Read AJ, Waljee AK, Saini SD (2020) A national survey of adoption of the 2018 American Cancer Society Colorectal Cancer Screening Guideline in Primary Care. Clin Gastroenterol Hepatol. https://doi.org/10.1016/j.cgh.2020.08.060

43. Montana Department of Public Health and Human Services (2020) Primary care provider survey: colorectal cancer screening knowledge and attitudes. Accessed 18 Nov 2020. [Online]. Available: http://www.dphhs.mt.gov/publichealth/cancer 